\title{
Hemiasterlin Analog E7974
}

National Cancer Institute

\section{Source}

National Cancer Institute. Hemiasterlin Analog E7974. NCI Thesaurus. Code C49083.

An analog of the sponge-derived anti-microtubule tripeptide hemiasterlin with antimitotic and potential antineoplastic activities. Hemiasterlin analog E7974 binds to the Vinca domain on tubulin, resulting in inhibition of tubulin polymerization and microtubule assembly; depolymerization of existing microtubules; inhibition of mitosis; and inhibition of cellular proliferation. This agent may have more affinity for the beta-3 tubulin isotype. 